\title{
Recursive Backstepping Stabilization of a Wheeled Mobile Robot
}

\author{
FAÏÇAL MNIF \\ Department of Electrical and Computer Engineering, Sultan Qaboos University, P.O. Box 33, Muscat 123, Muscat. \\ Oman. \\ On leave from the Institut National des Sciences Appliquees et de Technologie, Tunisia.
}

\begin{abstract}
This research is aimed to the development of a dynamic control to enhance the performance of the existing dynamic controllers for mobile robots. System dynamics of the car-like robot with nonholonomic constraints were employed. A Backstepping approach for the design of discontinuous state feedback controller is used for the design of the controller. It is shown that the origin of the closed loop system can be made exponentially stable. The control design is made on the basis of a suitable Lyapunov function candidate. The effectiveness of the proposed approach is tested trough simulation on a car-like vehicle mobile robot.
\end{abstract}

Key-Words: Backstepping. Mobile Robot. Chained form. Nonholonomic Systems.

\section{Introduction}

Wheeled mobile robots (WMRs) are increasingly present in industrial and service robotics, particularly when flexible motion capabilities are required on reasonably smooth ground and surfaces [1]. Several mobility configurations can be found in these applications. The most common are the tricycle and the car-like drive. Kinematics study of several configurations of WMRs can be found in [2].

Beside the relevance in industrial applications, the problem of autonomous motion planning and control of WMRs has attracted the interest of researchers in view of its theoretical challenges. In particular, these systems are typically examples of nonholonomic mechanical systems [3].

In the absence of workspace obstacles, the basic motion tasks assigned to a WMR may be reduced to moving between two postures and following a given trajectory. From a control viewpoint, the peculiar nature of nonholonomic kinematics makes the control problem easier than the first; in fact, it is known [4], that feedback stabilization at a given posture cannot be achieved via smooth time invariant control. This indicates that the problem is really nonlinear; linear control is ineffective, even locally, and innovative design is required.

The trajectory tracking problem of WMRs was globally solved in [5] by using nonlinear feedback control, and independently in [6] and [7] through the use of dynamic feedback linearization. Recursive backstepping control schemes for chained forms of WMRs have been also addressed by several authors [8].

It can be shown that the dynamic equations of a carlike vehicle mobile robot can be written in chained form as:

$$
\begin{aligned}
& \dot{x}_{1}=u_{1} \\
& \dot{x}_{1}=u_{2} \\
& \dot{x}_{1}=x_{2} u_{1} \\
& \vdots \\
& \dot{x}_{1}=x_{n-1} u_{1}
\end{aligned}
$$

where $\quad x=\left(x_{1}, x_{2}, \ldots, x_{n}\right)^{T} \in \mathfrak{R}^{n}$ denotes the state vector and $u=\left(u_{1}, u_{2}\right)^{T} \in \mathfrak{R}^{2}$.

Such a class of nonlinear systems can not be stabilized via continuous based time-invariant systems [9], which motivated the search of other stabilizing controls for this type of systems. Kolmanovsky and Mc-Clamroch [10] stated the art of existing solution for nonholonomic systems [10]. In the recent last few years many authors exhibit a particular attention to the design of discontinuous controller for chained systems, [11], [12].... In this paper we propose a systematic backstepping based procedure for the design of a discontinuous time-invariant controller for nonholonomic chained forms with application to nonholonomic mobile robot systems. It is shown that backstepping control for chained form systems can guarantee the boundedness of the whole state and makes the origin of the closed-loop system exponentially attractive provided that the initial states belong to the set defined by:

$\Omega_{0}: \Omega_{0}=\left\{\left(x_{1}(0), x_{2}(0), \ldots, x_{n}(0)\right)^{T} \in \mathfrak{R}^{n} / x_{1}(0) \neq 0\right\}$

The proposed control scheme is applied to the dynamics of a car-like vehicle mobile robot which investigation and reduction are presented exhaustively in section 2 . 


\section{The Car-like Vehicle}

In this section, we address reduction of the dynamic model of a car-like vehicle as shown in Figure 1. The dynamic model of a car is an example of underactuated nonholonomic systems with five degrees of freedom, two control inputs and two velocity constraints. Let $q=(x, y, \theta, \psi, \phi)$ denote the configuration vector of the system. $(x, y)$ denote the position of the center of the axle between the two rear wheels, $\theta$ is the orientation of the car body with respect to $\mathrm{x}$-axis, $\psi$ is the angle of rotation of each wheel and $\phi$ is the steering angle with respect the car body. The distance between the rear and front wheels axles is denoted by $l$.

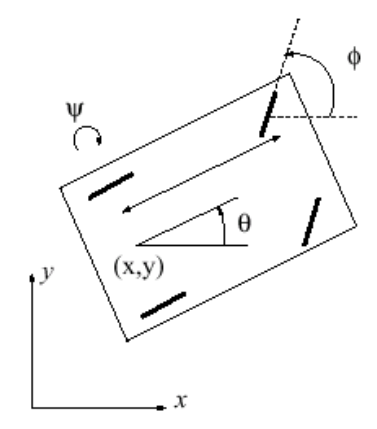

Fig.1. A car-like vehicle

The velocity constraints of the front and rear wheels are given by

$$
\begin{aligned}
& \sin (\theta+\psi) \frac{d}{d t}(x+l \cos \theta)-\cos (\theta+\phi) \frac{d}{d t}(x+l \sin \theta)=0 \\
& \sin (\theta) \dot{x}-\cos (\theta) \dot{y}=0
\end{aligned}
$$

or

$$
\begin{aligned}
& \sin (\theta+\psi) \dot{x}-\cos (\theta+\phi) \dot{y}-l \cos \phi \dot{\theta}=0 \\
& \sin (\theta) \dot{x}-\cos (\theta) \dot{y}=0
\end{aligned}
$$

which can be written as $W(q) \dot{q}=0$ where $W=\left(W_{1}, W_{2}\right)$ is partitioned according to $q_{x}=(x, y)$ and $q_{r}=(\theta, \psi, \phi)$ such that

$$
W_{1}(\theta, \phi)=\left[\begin{array}{cc}
\sin (\theta+\phi) & -\cos (\theta+\phi) \\
\sin (\theta) & -\cos (\theta)
\end{array}\right]
$$

and

$$
W_{2}(\theta, \phi)=\left[\begin{array}{ccc}
-l \cos \phi & 0 & 0 \\
0 & 0 & 0
\end{array}\right]
$$

Note that $W_{1}$ is not invertible.

Define now

$$
\omega_{12}(\theta, \phi)=-W_{1}^{-1} W_{2}=\left[\begin{array}{ccc}
\alpha_{1} & 0 & 0 \\
\alpha_{2} & 0 & 0
\end{array}\right]
$$

where

$$
\alpha_{1}(\theta, \phi)=\frac{l \cos \theta}{\tan \phi}, \alpha_{2}(\theta, \phi)=\frac{l \sin \theta}{\tan \phi} .
$$

And $D(q)$ can then be determined from $\omega_{12}(\theta, \phi)$ as

$$
D(q)=D(\theta, \phi)=\left[\begin{array}{ccc}
\alpha_{1} & 0 & 0 \\
\alpha_{2} & 0 & 0 \\
1 & 0 & 0 \\
0 & 1 & 0 \\
0 & 0 & 1
\end{array}\right]
$$

The Lagrangian of the dynamic car is given by

$$
\begin{aligned}
L= & \frac{1}{2} m\left[\left(\dot{x}-\frac{l}{2} \sin (\theta) \dot{\theta}\right)^{2}+\left(\dot{y}+\frac{l}{2} \cos (\theta) \dot{\theta}\right)^{2}+\right. \\
& \frac{1}{2}\left(J_{b}+2 J_{v}\right) \dot{\theta}^{2}+\frac{1}{2}\left[2 J_{h}\left(1+\frac{1}{\cos ^{2}(\phi)}\right)\right\} \dot{\psi}^{2}+\frac{1}{2} 2 J_{v}(\dot{\theta}+\dot{\phi})^{2}
\end{aligned}
$$

where $m$ is the mass of the car, $J_{\mathrm{b}}$ is the inertia of the body, $J_{\mathrm{h}}$ is the inertia of each wheel along the horizontal axes and $J_{\mathrm{v}}$ is the inertia of each wheel in the vertical axes. The Lagrangian can be expressed as

$$
L=\frac{1}{2} \dot{q}^{T}\left[\begin{array}{ccccc}
m & 0 & -(m l / 4) \sin \theta & 0 & 0 \\
0 & m & (m l / 4) \cos \theta & 0 & 0 \\
-(m l / 4) \sin \theta & (m l / 4) \cos \theta & J_{\theta} & 0 & 2 J_{v} \\
0 & 0 & 0 & 2 J_{h}\left(2+\tan ^{2}(\phi)\right) & 0 \\
0 & 0 & 2 J_{v} & 0 & 2 J_{v}
\end{array}\right] \dot{q}
$$

where $J_{\theta}=J_{b}+4 J_{v}+m l^{2} / 4$. The differential-algebraic equations of motion of the dynamic car are such as

$$
\frac{d}{d t} \frac{\partial L}{\partial \dot{q}}-\frac{\partial L}{\partial q}=W^{T}(q)\left[\begin{array}{l}
\lambda_{1} \\
\lambda_{2}
\end{array}\right]+\left[\begin{array}{l}
0_{3 \times 2} \\
I_{2 \times 2}
\end{array}\right]\left[\begin{array}{l}
u_{1} \\
u_{2}
\end{array}\right]
$$

where $\left(\lambda_{1}, \lambda_{2}\right) \in \mathfrak{R} \times \Re$ are the Lagrange multipliers and $\left(u_{1}, u_{2}\right) \in \mathfrak{R} \times \mathfrak{R}$ are the torques applied to the rear wheels and the steering wheel respectively. The dynamics of the car in (7) can be reduced to the cascade of the constraint equation and a reduced Lagrangian system with configuration vector $q=(\theta, \psi, \phi)$ as [13]

$$
M_{r}\left(q_{r}\right) \ddot{q}_{r}+C_{r}\left(q_{r}, \dot{q}_{r}\right) \dot{q}_{r}+G_{r}\left(q_{r}\right)=F_{r}\left(q_{r}\right) u
$$

where

$$
\begin{aligned}
& M_{r}=D^{T} M D \\
& C_{r}=D^{T} C D+D^{T} M \dot{D} \\
& F_{r}=D^{T} F
\end{aligned}
$$

By direct calculation and after simplification we get

$$
F_{r}=\left[\begin{array}{ll}
0 & 0 \\
1 & 0 \\
0 & 1
\end{array}\right]
$$

and

$$
M_{r}=M_{r}(\phi)=\left[\begin{array}{ccc}
\bar{J}_{\theta}(\phi) & 0 & 2 J_{v} \\
0 & 2 J_{h}\left(2+\tan ^{2}(\phi)\right) & 0 \\
2 J_{v} & 0 & 2 J_{v}
\end{array}\right]
$$

with

$$
\bar{J}_{\theta}(\phi)=J_{\theta}+\frac{m l^{2}}{\tan ^{2}(\phi)}
$$

Clearly the reduced Lagrangian is itself underactuated with three degrees of freedom and two controls. In addition, $(\theta, \psi)$ are the external variables and $\phi$ is the 
shape variable of the car. The dynamics of the actuated variables $(\theta, \psi)$ of the reduced system can be linearized as

$$
\begin{aligned}
& \ddot{\psi}=\tau_{1} \\
& \ddot{\phi}=\tau_{2}
\end{aligned}
$$

using an explicit collocated change of variable in the form

$$
u=\eta(\phi)+\beta\left(\phi, \dot{q}_{r}\right)
$$

where

$$
\eta(\phi)=\left[\begin{array}{cc}
2 J_{h}\left(2+\tan ^{2}(\phi)\right) & 0 \\
0 & 2 J_{v}\left(1-\frac{2 J_{v} \tan ^{2}(\phi)}{m l^{2} \tan ^{2}(\phi)}\right.
\end{array}\right]
$$

$\eta(\phi)$ is well defined and positive definite for all $\phi$.

From the first constraint equation, we can solve for $\dot{\theta}$ to get

$$
\dot{\theta}=\tan (\phi)(\cos (\theta) \dot{x}+\sin (\theta) \dot{y})
$$

From this equation, the overall dynamics of the car can be written as

$$
\begin{aligned}
& \dot{x}=r \cos \theta \dot{\psi} \\
& \dot{y}=r \sin \theta \cdot \dot{\psi} \\
& \dot{\theta}=\frac{r}{l} \tan \theta \cdot \dot{\psi} \\
& \ddot{\psi}=\tau_{1} \\
& \ddot{\phi}=\tau_{2}
\end{aligned}
$$

where $r$ is the radius of the wheel.

After normalization of the units of $(x, y)$ by $r$, and taking $\dot{\psi}=\omega_{1}$ we get

$$
\begin{aligned}
& \dot{x}=r \cos \theta \cdot \omega_{1} \\
& \dot{y}=r \sin \theta \cdot \omega_{1} \\
& \dot{\theta}=\frac{1}{l} \tan \theta \cdot \omega_{1} \\
& \dot{\phi}=\omega_{2}
\end{aligned}
$$

where

$$
\dot{\omega}_{1}=\tau_{1}, \dot{\omega}_{2}=\tau_{2}
$$

Now applying the change of coordinates and control as:

$$
\begin{aligned}
& x_{1}=x \\
& x_{2}=\frac{\tan \phi}{l \cos ^{3} \theta} \\
& x_{3}=\tan \theta \\
& x_{4}=y \\
& u_{1}=\cos \theta \cdot \omega_{1} \phi \\
& u_{2}=\frac{1+\tan ^{2} \phi}{l \cos ^{3} \theta} \omega_{2}+\frac{3 \tan \theta \tan ^{2} \phi}{l^{2} \cos ^{3} \theta}
\end{aligned}
$$

transforms finally the nonholonomic system (13) into a chained form-type system of the form

$$
\begin{aligned}
& \dot{x}_{1}=u_{1} \\
& \dot{x}_{2}=u_{2} \\
& \dot{x}_{3}=x_{2} u_{1} \\
& \dot{x}_{4}=x_{3} u_{1}
\end{aligned}
$$

\section{Backstepping control of the car-like vehicle}

Control design for broad classes of nonholonomic mechanical systems can be reduced to the control of chained -type systems described by.

$$
\begin{aligned}
& \dot{x}_{1}=u_{1} \\
& \dot{x}_{1}=u_{2} \\
& \dot{x}_{1}=x_{2} u_{1} \\
& \vdots \\
& \dot{x}_{1}=x_{n-1} u_{1}
\end{aligned}
$$

To guarantee the exponential convergence of $x_{1}$, we use a state feedback such that

$$
u_{1}=-k x_{1}, k>0 \text {, }
$$

and then

which gives

$$
\dot{x}_{1}=-k x_{1}
$$

$$
x_{1}=c e^{-k t} .
$$

Under this control, the system (15) becomes:

$$
\begin{aligned}
& \dot{x}_{1}=-k x_{1} \\
& \dot{x}_{1}=u_{2} \\
& \dot{x}_{1}=-k x_{1} x_{2} \\
& \vdots \\
& \dot{x}_{1}=-k x_{1} x_{n-1}
\end{aligned}
$$

Now the problem consists of finding a control law $u_{2}$ such that, if the initial state belongs to the $\operatorname{set} \Omega=\left\{\left(x_{1}(0), x_{2}(0), \ldots x_{n}(0)\right)^{T} \in R^{n} / x_{1}(0) \neq 0\right\}$, the whole state of the closed loop converges exponentially to zero.

As the backstepping procedure requires that the design starts from the $\mathrm{n}^{\text {th }}$ coordinates and going forth ward to the first state therefore, for the clarity of the procedure a change of coordinate transformation has been made such that :

$$
z_{i}=x_{n-i+1}, \quad 1 \leq i \leq n,
$$

and then (46) becomes 


$$
\begin{aligned}
& \dot{z}_{1}=z_{2} u_{1} \\
& z_{2}=z_{3} u_{1} \\
& \vdots \\
& z_{n-1}=u_{2} \\
& \dot{z}_{n}=u_{1}
\end{aligned}
$$

Substituting $u_{1}$ in (19) one gets

$$
\begin{aligned}
& \dot{z}_{1}=-k z_{n} z_{2} \\
& \dot{z}_{2}=-k z_{n} z_{3} \\
& \vdots \\
& \dot{z}_{n}=-k z_{n}
\end{aligned}
$$

the design procedure is made up of $(n-1)$ steps, consists of making the $(n-1)^{\text {th }}$ states converge exponentially to zero.

\section{Step1}

Consider the first equation of the system (20) and the Lyapunov function candidate:

$$
V_{1}=\frac{1}{2 k} z_{1}^{2}
$$

The time derivative of $V_{1}$ gives

$$
\dot{V}_{1}=\frac{1}{k} z_{1} \dot{z}_{1}=-z_{2} z_{n}
$$

by tacking $z_{2}=-k_{1} \frac{z_{2}}{z_{n}} ; \quad k_{1}>0$

Equation (22) becomes:

$$
\dot{V}_{1}=-k_{1} z_{2}^{2}=-2 k k_{1} V_{1}
$$

which by integration gives

$$
V_{1}=\beta e^{-2 k k_{1} t}
$$

$V_{1}$ decays to zero as $t \rightarrow \infty$ since $k k_{1}>0$.

\section{Step 2}

We introduce a new variable $\varepsilon_{2}=z_{2}-k_{1} \frac{z_{2}}{z_{n}}$, this can be explained as a deviation between the real state $z_{2}$ and the desired virtual control

Hence we get

$$
\alpha_{1}=k_{1} \frac{z_{2}}{z_{n}}=\left(z_{2}\right)_{d} .
$$

$$
\begin{aligned}
& \dot{\varepsilon}=-k z_{3} z_{n}-\dot{\alpha} 1 \\
& z_{1}=-k z_{2} z_{n}=-k\left(\varepsilon_{2}+\alpha_{1}\right) z_{n}
\end{aligned}
$$

Consider now the Lyapunov function candidate:

$$
V_{2}=V_{1}+\frac{1}{2 k} \varepsilon_{2}^{2}=\frac{1}{2 k} z_{1}^{2}+\frac{1}{2 k} \varepsilon_{2}^{2}
$$

The time derivative of (26) is then

$$
\dot{V}_{2}=-z_{1} z_{n}\left(\varepsilon_{2}+\alpha_{1}\right)-\left(z_{3} z_{n}+\frac{\alpha_{1}}{k}\right) \varepsilon_{2}
$$

$$
\dot{V}_{2}=\dot{V}_{1}-\varepsilon_{2}\left(z_{3} z_{n}+\frac{\dot{\alpha}}{k}\right)-z_{1} z_{n} \varepsilon_{2}
$$

By choosing

$$
\alpha_{2}=\left(z_{2}\right)_{d}=-z_{1}+\frac{k_{2} \varepsilon_{2}^{2}}{z_{n}}-\frac{\alpha_{1}}{k z_{n}} ; k_{2}>0
$$

then $\dot{V}_{2}$ is negative definite.

\section{step 3}

Likewise in step 2 we introduce a new variable $\varepsilon_{3}$ such that :

$$
\varepsilon_{3}=z_{3}-\alpha_{2}
$$

we have therefore

$$
\begin{aligned}
& \dot{\varepsilon}_{2}=-k\left(\varepsilon_{3}+\alpha_{2}\right) z_{n}-\dot{\alpha}_{1} \\
& \dot{\varepsilon_{3}}=-k z_{4} z_{n}-\dot{\alpha}_{2}
\end{aligned}
$$

Consider the Lyapunov function candidate as

$$
V_{3}=\frac{1}{2 k} z_{1}^{2}+\frac{1}{2 k} \sum_{i=2}^{3} \varepsilon_{i}^{2}
$$

The time derivative of (31) gives:

$$
\begin{aligned}
\dot{V}_{3}= & -z_{1} z_{n}\left(\alpha_{1}+\varepsilon_{2}\right)+\frac{\varepsilon_{2}}{k}\left(-k\left(\varepsilon_{3}+\alpha_{2}\right) z_{n}-\dot{\alpha}_{1}\right) \\
& +\frac{\varepsilon_{3}}{k}\left(-k z_{4} z_{n}-\dot{\alpha} 2\right) \\
& =\dot{V}_{2}-\varepsilon_{3} z_{n} \varepsilon_{2}+\frac{\varepsilon_{3}}{k}\left(-k z_{4} z_{n}-\dot{\alpha}_{2}\right)
\end{aligned}
$$

Taking the new variable $\alpha_{3}$ as :

$$
\alpha_{3}=\left(z_{4}\right)_{d}=-\varepsilon_{2}+k_{3} \frac{\varepsilon_{3}}{z_{n}}-\frac{\alpha_{2}}{k z_{n}}
$$

the $\dot{V}_{3}$ is negative definite.

Recursively for $i=2 \cdots n-2$ the developed procedure can be generalized by taking $\varepsilon_{i}=z_{i}-\alpha_{i-1}$ and considering the Lyapunov function candidate:

$$
\dot{V}_{i}=\dot{V}_{i-1}-\varepsilon_{i} z_{n} \varepsilon_{i-1}+\frac{\varepsilon_{i}}{k}\left(-k z_{i+1} z_{n}-\dot{\alpha}_{i-1}\right)
$$


with $\alpha_{i}$ as

$$
\alpha_{i}=\left(z_{i+1}\right)_{d}=-\varepsilon_{i-1}+\frac{k_{i} \varepsilon_{i}}{z_{n}}-\frac{\dot{\alpha}}{k z_{n}}
$$

The time derivative of (35) can be obtained as

$$
\dot{V}_{i}=-k_{1} y_{1}^{2}-\sum_{j=2}^{i} k_{j} z_{j}^{2} \quad k_{i}>0, \quad 2 \leq i \leq n-2
$$

\section{Step n-1}

Same as in step $n$-1, we assign as a new variable the deviation between $z_{n-1}$ and the new $\operatorname{control} \alpha_{n-2}$.

Introduce the Lyapunov function candidate:

$$
V_{n-1}=V_{n-2}+\frac{1}{2 k} \varepsilon_{n-1}^{2}
$$

the time derivative of $V_{\mathrm{n}-1}$ is given by

$$
\dot{V}_{n-1}=\dot{V}_{n-2}-\varepsilon_{n-2} \varepsilon_{n-1} z_{n}+\frac{\varepsilon_{n-1}}{k}\left(u_{2}-\dot{\alpha}_{n-2}\right)
$$

Under the following control $u_{2}$, defined over $\Omega$,

$$
u_{2}=k \varepsilon_{n-2} z_{n}-k k_{n-1} \varepsilon_{n-1}+\dot{\alpha}_{n-2}
$$

Equation (38) becomes:

$$
\dot{V}_{n-1}=\dot{V}_{n-2}-k_{n-1} \varepsilon_{n-1}^{2}
$$

which is negative definite for $k_{n-1}>0$.

The above development can be summarized in the following theorem

\section{Theorem 1}

Consider the system (15) with the control law

$$
\begin{gathered}
u_{1}= \begin{cases}-k x_{1} & \text { for } x_{1}(0) \neq 0 \\
u_{1}^{*} & \text { for } x_{1}(0)=0\end{cases} \\
u_{2}= \begin{cases}k \varepsilon_{n-2} z_{n}-k k_{n-1} \varepsilon_{n-1}+\alpha_{n-2} & \text { for } x_{1}(0) \neq 0 \\
u_{2}^{*} & \text { for } x_{1}(0) \neq 0\end{cases}
\end{gathered}
$$

where $u_{1}^{*} \in \mathfrak{R} /\{0\}, \quad u_{2}^{*} \in \mathfrak{R} /\{0\}$ are two constant values and where

$$
\alpha_{i}=\left(z_{i+1}\right)_{d}=-\varepsilon_{i-1}+\frac{k_{i} \varepsilon_{i}}{z_{n}}-\frac{\dot{\alpha}_{i-1}}{k z_{n}}
$$

with $k>0$, and $k_{i}>0, i=1, \cdots, n-1$

then

i) the whole state $x=\left(x_{1}, \ldots, x_{n}\right)^{T}$ is bounded and decays exponentially to zero when $t \rightarrow \infty$,

ii) the control law is well defined and bounded for all $t \geq 0$.

the control law to apply to guarantee that the closed loop system is exponentially stable hence can be deduced by assigning the control $u_{1}$ and $u_{2}$ the expressions $u_{1}=-k x_{1}$ and $u_{2}$ as in (39) respectively.

\section{Simulation Results}

The technique presented in this paper is tested on the system

$$
\begin{aligned}
& \dot{x}=v \cos \theta \\
& \dot{y}=v \sin \theta \\
& \dot{\theta}=\frac{v}{l} \tan \theta \\
& \dot{\phi}=\omega
\end{aligned}
$$

where $v$ is the constant linear velocity of the car and 1 is the distance between the front and rear wheels axles. Results are depicted in Figures 1-5. The Initial conditions are taken as

$$
x(0)=\left[x_{0}, y_{0}, \theta_{0}, \varphi_{0}\right]^{T}=\left[1.5,2, \frac{\pi}{4},-\frac{\pi}{2}\right]^{T}
$$

and the following gains are used

$$
k_{1}=3, k_{2}=2, k_{3}=0.5 \text { and } k_{4}=0.5 \text {. }
$$

Figure 2 shows the path traversed by the mobile robot. Figures 3-6 show the time behavior of the variable, $x$, $y, \theta$ and $\phi$. It is clear that the discontinuous backstepping approach presented in this paper guarantees the convergence and the exponential stabilization of all the states of the system.

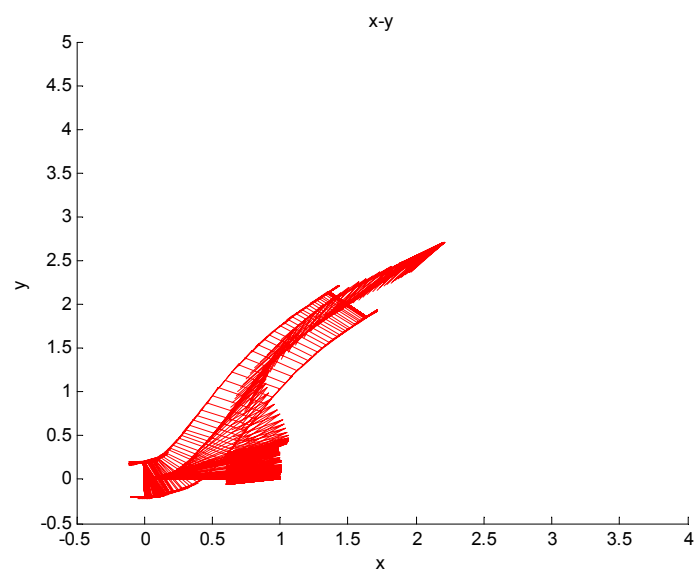

Fig. 2. Path to the origin of the mobile robot

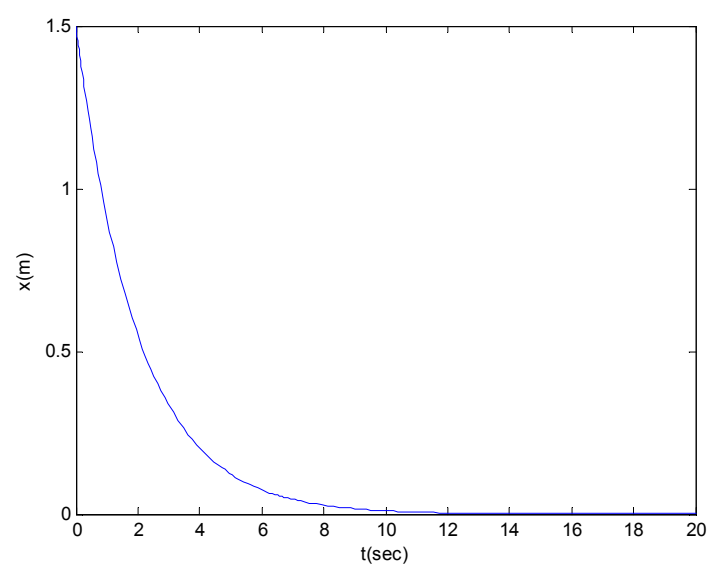

Fig. 3 Time history of the variable $x$. 


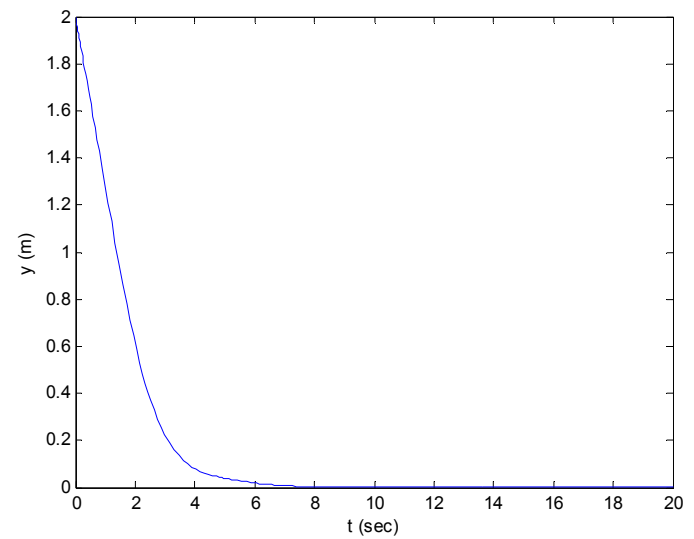

Fig. 4. Time history of the variable $y$.

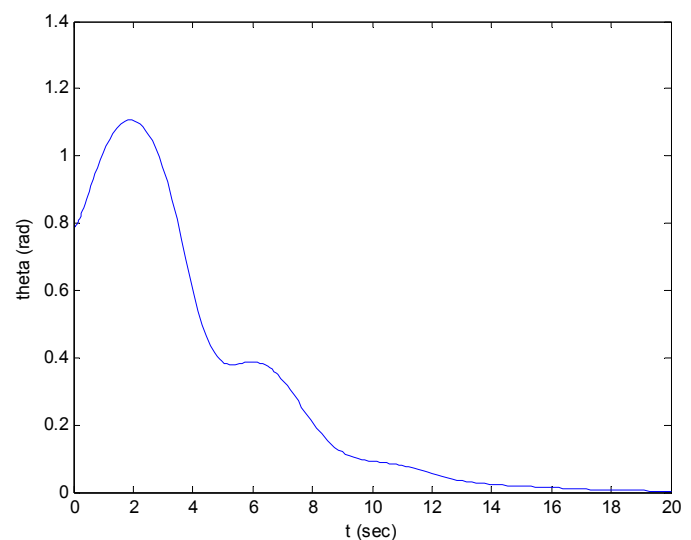

Fig. 5 Time history of the variable $\theta$

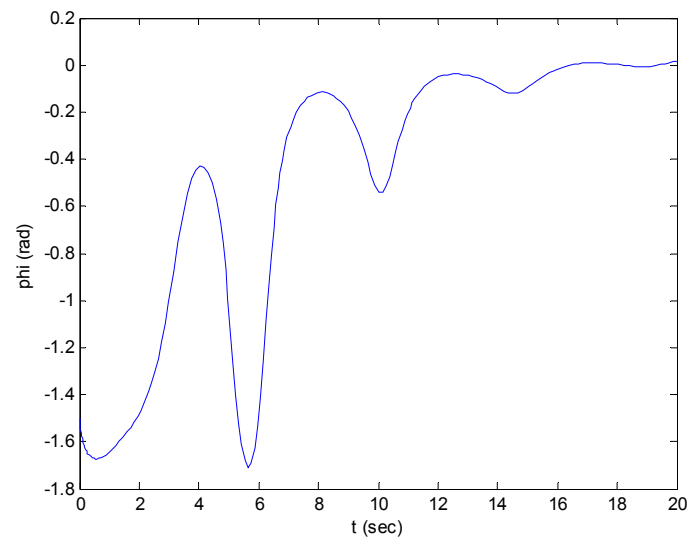

Fig. 6 Time history of the variable $\phi$.

\section{Conclusions}

The paper presents a novel technique for backstepping discontinuous control with application to the stabilization of a nonholonomic mobile robot. The methodology is not restricted, though to nonholonomic systems but can be applied to a broad class of strictly feedback discontinuous systems. The proposed control scheme is smooth everywhere except at $x_{1}=0$. The discontinuity involved in the control is not very restrictive since it occurs just for $x_{1}(0)=0$. The simulation results made on a car-like mobile robot demonstrate the validity of the proposed control.

\section{References}

[1] Schraft, R.D. and Schmierer, G., "Serviceroboter", Springer Verlag, 1998.

[2] Alexander, J.C., and Maddocks, J.H., "On the Kinematics of wheeled mobile robot", International Journal of Robotics Research, 8, 5, pp. 15-27, 1989.

[3] Neimark, J.I., and Fufaev, F.A., "Dynamics of Nonholonomic Systems", American mathematical Society, Providence, RI. 1972.

[4] Campion, G., d'Andrea-Novel, B, and Bastin, G., "Modeling and state feedback control of nonholonomic mechanical systems", Proc. of the $30^{\text {th }}$ IEEE Conference on Decision and Control, Brighton, UK, pp. 1184-1189, 1991.

[5] Samson, C., and Ait-Abderrahim, K., "Feedback control of a nonholonomic wheeled cart in Cartesian space", Proc. of the IEEE International Conference on Robotics and Automation, Sacramento, CA, pp. 11361141, 1991.

[6] De Luca, A. and Di Benedetto, M.D., "Control of nonholonomic systems via dynamic compensation ", Kybernetica, 29, 6, pp. 1993.

[7] d'Andrea-Novel, B., Bastin, Campion, G., "Control of nonholonomic wheeled mobile robots by state feedback linearization", International Journal of Robotics Research, 14, 6, pp. 543-559, 1995.

[8] Jiang, Z-P, Nijimer, H., "A recursive technique for tracking control of nonholonomic systems in chained form", IEEE Transactions on Automatic Control, 44, 2, pp. 265-279, 1999.

[9] R.W. Brockett, "Asymptotic stability and feedback stabilization", Progress in Math., vol. 27, Birkhauser, pp. 181-208, 1993.

[10] I. Klomanovsky and N.H., Mc-Clamroch, "Developments in nonholonomic control problems", IEEE Control System magazine, 15, 6, pp. 20-36, 1995.

[11] A. Tayebi, M. Tadjine and A. Rachid, "Discontinuous control design for the stabilization of nonholonomic systems in chained form using the backstepping approach", Proc of the $36^{\text {th }}$ IEEE CDC, pp. 3089-3090, 1997.

[12] H. G. Tanner and K. Kyriakopoulos, "Discontinuous Backstepping for stabilization of nonholonomic mobile robots", Proc of the IEEE ICRA, pp. 3948-3953, 2002.

[13] F. Mnif, "On the reduction and Control of a class of nonholonomic underactuated systems", Journal of Electrical Engineering, 54, 1-2, pp. 22-29, 2003. 